\title{
Lentivirus-mediated RNA interference targeting UbcH10 reduces cell growth and invasion of human osteosarcoma cells via inhibition of Ki-67 and matrix metalloproteinases
}

\author{
SHAN-TAO WANG ${ }^{1,2 *}$, DAN-ZHI LI ${ }^{2 *}$, JIAN-MIN LI $^{1}$, JUN FANG $^{2}$, \\ HUA-ZHUANG $\mathrm{LI}^{2}$, PEI-JIAN TONG ${ }^{3}$ and FU-CUN LIU ${ }^{3}$
}

\begin{abstract}
${ }^{1}$ Department of Orthopedics, Qilu Hospital, Shandong University, Jinan, Shandong 510012; ${ }^{2}$ Department of Orthopedics, Yidu Central Hospital of Weifang, Qingzhou, Shandong 262500; ${ }^{3}$ Department of Orthopedics, The First Affiliated Hospital of Zhejiang University of Traditional Chinese Medicine, Hangzhou, Zhejiang 310006, P.R. China
\end{abstract}

Received July 3, 2014; Accepted February 10, 2015

DOI: $10.3892 / \mathrm{ol} .2015 .3023$

\begin{abstract}
Osteosarcoma (OS) is the most commonly diagnosed primary malignancy affecting the bone. UbcH10 is a cancer-related E2-ubiquitin-conjugating enzyme. An overexpression of UbcH10 is significantly associated with tumor grade and cellular proliferation. However, limited evidence exists with regard to the biological function of UbcH10 in OS. The present study created a UbcH10 knockdown OS cell line using lentivirus-mediated RNA interference. The expression of $\mathrm{UbcH} 10$ was significantly reduced in $\mathrm{UbcH} 10$-targeted small hairpin RNA-expressing lentivirus OS cells. The downregulation of UbcH10 suppressed OS cell proliferation and colony formation ability via decreased Ki-67 expression. UbcH10 knockdown OS cells exhibited impaired invasion and migration abilities. Furthermore, knockdown of UbcH10 led to decreased levels of matrix metalloproteinase- 3 and -9 in OS cells. The present study demonstrated the role of UbcH10 in OS cell proliferation, invasion and migration, which suggests that UbcH10 may be a potential candidate for OS therapy.
\end{abstract}

\section{Introduction}

Osteosarcoma (OS) is the most commonly diagnosed primary malignant cancer affecting the bone, and one of the most

Correspondence to: Dr Fu-Cun Liu, Department of Orthopedics, The First Affiliated Hospital of Zhejiang University of Traditional Chinese Medicine, 54 Youdian Road, Hangzhou, Zhejiang 310006, P.R. China

E-mail: dxwzliu@126.com

*Contributed equally

Key words: UbcH10, matrix metalloproteinases, osteosarcoma, growth, invasion, Ki-67 heterogeneous types of human tumor $(1,2)$. The incidence rates of OS are 1.7 per million and 4.2 per million, in individuals aged 25-59 years old and $\geq 60$ years old, respectively (3). OS predominantly occurs in children and young adolescents, and is characterized by a high level of malignancy, relapse and metastasis, and poor prognoses. In recent years, the combination of systemic chemotherapy and modern surgery has improved the treatment of OS. However, no substantial improvement in patient survival has been observed (4) and the five-year survival rate for patients with metastatic OS is $20-30 \%$ (3). Therefore, it is important to understand the molecular mechanisms underlying OS in order to improve treatment strategies. In addition, the identification and characterization of molecules involved in OS tumorigenesis is required to aid advances in therapeutic strategies.

Ubiquitin-conjugating enzyme E2 C (UbcH10) belongs to the E2 family and is involved in ubiquitin-dependent proteolysis $(5,6)$. UbcH10 is highly conserved and consists of a core domain with a catalytic Cys residue, and an $\mathrm{N}$-terminal extension (7). The core domain interacts with a ubiquitin-fold domain in the E1 enzyme to form a ubiquitin adduct, and the $\mathrm{N}$-terminal extension regulates E3 enzyme activity. Previous studies have demonstrated that $\mathrm{UbcH} 10$ is crucial in mitotic regulation, and is required for the degradation of mitotic checkpoint proteins, cyclins $(5,8)$ and other mitosis-related substrates $(7,9)$, which are essential for spindle assembly checkpoints and mitotic exits. Increasing evidence has indicated that $\mathrm{UbcH} 10$ is abnormally overexpressed in a number of malignant tumors, including cancers of the adrenal gland, bladder, brain, breast, cervix, colon, rectum, esophagus, liver, lung, nasopharynx, ovary, prostate (late-stage), pancreas, stomach, thyroid and uterus $(10,11)$. UbcH10 is recognized as a potential cancer biomarker (11). An overexpression of $\mathrm{UbcH} 10$ is significantly associated with the pathological grading of tumors, high cellular proliferation and poor prognoses of cancers affecting the adrenal gland, breast, colon, liver, lung and ovary $(10,11)$. Furthermore, UbcH10 transgenic mice are prone to developing a range of spontaneous and carcinogen-induced tumors (12). By contrast, silencing of UbcH10 inhibits glioma and colorectal cancer 
proliferation $(13,14)$. However, limited evidence exists regarding the biological function of UbcH10 in OS.

In the present study, UbcH10 was knocked down in the OS U2OS and SaOS2 cell lines through lentivirus-mediated RNA interference (RNAi). The role of UbcH10 in OS progression was then analyzed in vitro. The cellular proliferation, invasion, colony formation and migration abilities were determined in $\mathrm{UbcH} 10$ knockdown cells. In addition, the expression of Ki-67 and matrix metalloproteinases (MMPs) were analyzed.

\section{Materials and methods}

Cell culture. The human OS U2OS and SaOS2 cell lines were obtained from the Institute of Biochemistry and Cell Biology (Shanghai, China). The cells were incubated in RPMI-1640 medium supplemented with $10 \%$ fetal calf serum (Thermo Fisher Scientific Inc., Waltham, MA, USA) and 1\% antibiotics (penicillin and streptomycin; Sigma-Aldrich, St. Louis, MO, USA) at $37^{\circ} \mathrm{C}$ in a humidified $5 \% \mathrm{CO}_{2}$ atmosphere.

Lentivirus-mediated short hairpin RNA (shRNA) transfection. The shRNA oligos of UbcH10 were designed according to its sequence in the NCBI database as follows: 5'-AACCUGCAAGAAACCUACUCA-dTdT-3'. The sequence of the control shRNA was as follows: 5'-AAAUGCACACACACAUACUCG-dTdT-3'. The fragments of shRNA were inserted into the lentivirus vector and transfected into HEK293 cells with packaging vectors using Lipofectamine 2000 (Life Technologies, Grand Island, NY, USA). After $48 \mathrm{~h}$, the recombinant lentivirus was collected from the media for further infection.

The U2OS and $\mathrm{SaOS} 2$ cells were cultured in a 6-well plate at a density of $12 \times 10^{4}$ cells per well. Subsequent to a 24 -h culture, the cells were transfected with the recombinant lentivirus at a multiplicity of infection of 20 . At $48 \mathrm{~h}$ post-infection, the cells were observed using a fluorescence microscope (DM IL LED; Leica Microsystems, Wetzlar, Germany). The infection efficiencies were determined by the ratio of green fluorescent protein (GFP)-positive cells to total cells.

Western blot analysis. At 3 days post lentiviral infection, the U2OS and SaOS2 cells were collected and lysed in RIPA buffer $(150 \mathrm{mM} \mathrm{NaCl}, 100 \mathrm{mM}$ Tris- $\mathrm{HCl}, 1 \%$ Tween-20, $1 \%$ sodium deoxycholate and $0.1 \%$ SDS) supplemented with $1 \mathrm{mM}$ phenylmethylsulfonyl fluoride (PMSF) and a protease inhibitor cocktail. Following centrifugation at $13,000 \mathrm{x} \mathrm{g}$ for $15 \mathrm{~min}$, the supernatant was collected and boiled with $2 X$ SDS protein sample buffer. The proteins were separated using SDS-PAGE and transferred to polyvinylidene fluoride membranes. The membranes were blocked with Tris-buffered saline and Tween 20 (TBST; Beijing SolarBio Science \& Technology Co., Ltd., Beijing, China) plus $1 \%$ bovine serum albumin (Westang Bio-Tech Co., Ltd., Shanghai, China) for $1 \mathrm{~h}$ and probed with a variety of antibodies overnight at $4^{\circ} \mathrm{C}$. Next, the membranes were washed with TBST for $15 \mathrm{~min}$ and probed with horseradish peroxidase-conjugated secondary antibodies for $1 \mathrm{~h}$. The membranes were then washed with TBST for $15 \mathrm{~min}$ and signals were detected by enhanced chemiluminescence using SuperSignal West Pico Chemiluminescent Substrate (Thermo Fisher Scientific Inc.) and the Amersham Imager 600 (GE Healthcare, Pittsburgh, PA, USA). The primary antibodies used in the present study were: Anti-UbcH10 (1:500; cat. no. 14234S; Cell Signaling Technology, Inc., Danvers, MA, USA), anti-GAPDH (1:10,000; cat. no. sc-365062; Santa Cruz Biotechnology, Inc., Dallas, TX, USA), anti-Ki-67 (1:1,000; cat. no. sc-7846; Santa Cruz Biotechnology, Inc.), anti-MMP-3 (1:1,000; cat. no. 14351S; Cell Signaling Technology, Inc.) and anti-MMP-9 (1:1,000; cat. no. sc-21733; Santa Cruz Biotechnology, Inc.). The secondary antibodies were horseradish peroxidase-conjugated goat anti-rabbit immunoglobulin G (1:2,000; cat. no. sc-2004; Santa Cruz Biotechnology, Inc.).

MTT assay. In brief, the U2OS and $\mathrm{SaOS} 2$ cells were cultured in a 96-well plate at a density of $10^{4}$ cells per well. Subsequent to a 24-h incubation, the cells were transfected with a recombinant lentivirus carrying shRNA. At various time-points of 1, 2, 3, 4 and 5 days, MTT (Sigma-Aldrich) was added at a final concentration of $5 \mathrm{mg} / \mathrm{ml}$ and incubated with the cells at $37^{\circ} \mathrm{C}$ for $4 \mathrm{~h}$. After removing the medium, dimethyl sulfoxide was added in order to terminate the reaction. All wells were analyzed using an ELISA reader (Bio-Rad Laboratories, Inc., Hercules, CA, USA) at a wavelength of $490 \mathrm{~nm}$.

Colony formation assay. After 5 days of lentivirus treatment with control shRNA-expressing lentivirus (Lv-shCon) or UbcH10-targeted shRNA-expressing lentivirus (Lv-shUbcH10), the U2OS and SaOS2 cells were trypsinized, counted and then replated in a 6-well plate at a concentration of 200 cells per well. The cell samples were then allowed to grow for 14 days in order to form natural colonies. Following this, the plate was washed twice with phosphate-buffered saline solution and stained for 10 min with Giemsa (Sigma-Aldrich). Images of the stained colonies were then captured under a fluorescence microscope. Finally, the total number of colonies (N50 cells/colony) and the total number of cells in each colony were counted and analyzed.

Transwell invasion assay. The invasion of U2OS and SaOS2 cells was analyzed using BioCoat Transwell chambers (Corning Incorporated, Corning, New York, NY, USA). The cells were serum starved for $24 \mathrm{~h}$ and then harvested. In total, $2 \times 10^{4}$ cells were added to the upper chamber, which was coated with Matrigel ${ }^{\mathrm{TM}}$ matrix (BD Biosciences, Franklin Lakes, NJ, USA). Subsequent to a $24-\mathrm{h}$ incubation at $37^{\circ} \mathrm{C}$ in a humidified atmosphere of $5 \% \mathrm{CO}_{2}$, the cells on the upper surface of the chamber were removed with a cotton swab. The cells that had invaded to the bottom surface of the insert were fixed with $70 \%$ ethanol and stained with crystal violet. The invasiveness was quantitated by counting the number of cells on five different random views using a light microscope (DMil; Leica Microsystems) (magnification, x10). All experiments were repeated at least three times, with more than three wells for each treatment.

Migration assay. Cellular migration was analyzed using a wound healing assay. The U2OS and SaOS2 cells were cultured to $90-95 \%$ confluence and then serum starved for $24 \mathrm{~h}$. Following this, the monolayers of cells were carefully wounded using sterilized pipette tips. The wound closure was determined using a light microscope, and images were captured at the indicated time points. 
A

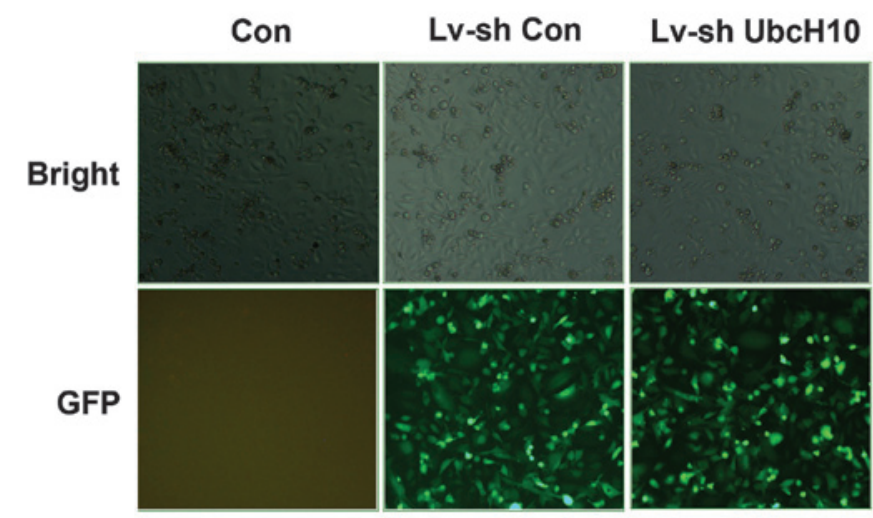

B

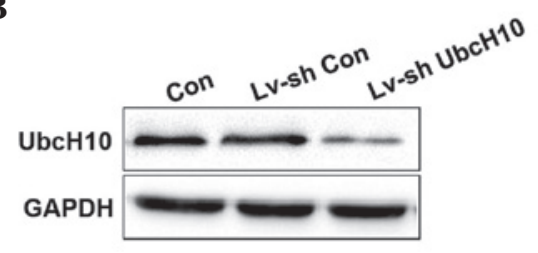

D

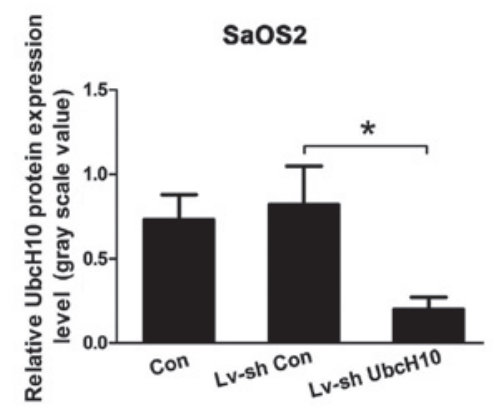

C

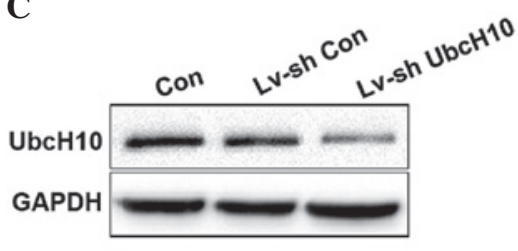

$\mathbf{E}$

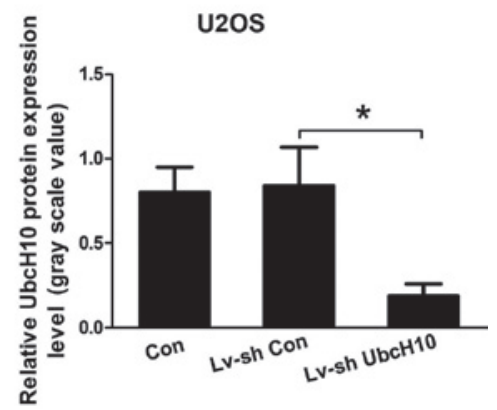

Figure 1. UbcH10 expression is suppressed in osteosarcoma cells by lentivirus-mediated RNA interference. (A) Representative images of Con, and Lv-shConand Lv-shUbcH10-transfected OS cells captured under a fluorescence microscope. Upper panel, bright field; lower panel, GFP. Western blotting analysis revealing the protein levels of UbcH10 in Con, and Lv-shCon- and Lv-shUbcH10-transfected (B) SaOS2 cells and (C) U2OS cells. GAPDH was used as a control protein. Calculation of UbcH10 protein levels in (D) SaOS2 cells and (E) U2OS cells (normalized to GAPDH). *P<0.05 vs. the control group. Con, control; Lv-shCon, control small hairpin RNA-expressing lentivirus; Lv-shUbcH10, UbcH10-targeted small hairpin RNA-expressing lentivirus; GFP, green fluorescent protein.

Statistical analysis. The cell culture experiments were performed in triplicate. Student's $t$-test was used to analyze the significance of differences. Two-tailed $\mathrm{P}<0.05$ was considered to indicate a statistically significant difference, and the data are presented as the mean \pm standard deviation.

\section{Results}

Lentivirus-mediated RNAi efficiently suppresses the expression of $\mathrm{UbcH} 10$ in $\mathrm{OS}$ cells. At $24 \mathrm{~h}$ post lentivirus-transfection, $>90 \% \mathrm{Lv}$-shCon and $\mathrm{Lv}$-ShUbcH10 transfected U2OS and SaOS2 cells exhibited GFP-positive signals (Fig. 1A), which indicated that the recombinant lentivirus was able to infect the OS cells with high efficiency. The proportion of positive cells that were transfected with $\mathrm{Lv}$-shUbcH10 was $>90 \%$, as evidenced by GFP expression 3 days after transfection (Fig. 1A). Further western blot analysis revealed that the protein levels of $\mathrm{UbcH} 10$ were significantly reduced in Lv-shUbcH10-transfected U2OS and SaOS2 cells $(\mathrm{P}=0.018$ and $\mathrm{P}=0.021$, respectively; Fig. 1B-E). The control shRNA did not affect the expression of UbcH10. These data demonstrate the high gene transfer efficiency of lentiviruses in OS cells, and suggest that the expression of UbcH10 is efficiently knocked down by shUbcH10.

UbcH10-targeted RNAi reduces OS cell proliferation and colony formation. Proliferation is a key process involved in the progression of tumors. In order to determine whether shUbcH10 has an inhibitory effect upon OS cell growth, an MTT assay was performed. As shown in Fig. $2 \mathrm{~A}$ and B, the growth curves for UbcH10 knockdown U2OS and SaOS2 cells were significantly lower during the 5-day incubation than those for control cells and Lv-shCon-transfected cells $(\mathrm{P}=0.0017$ and $\mathrm{P}=0.0028$, respectively). The colony formation assay demonstrated that the colony numbers of $\mathrm{Lv}$-shUbcH10-transfected U2OS and $\mathrm{SaOS} 2$ cells were significantly reduced compared with those of the control cells and Lv-shCon-transfected cells $(\mathrm{P}=0.0015$ and $\mathrm{P}=0.0022$, respectively) (Fig. $2 \mathrm{C}$ and D). This indicates that the 
A

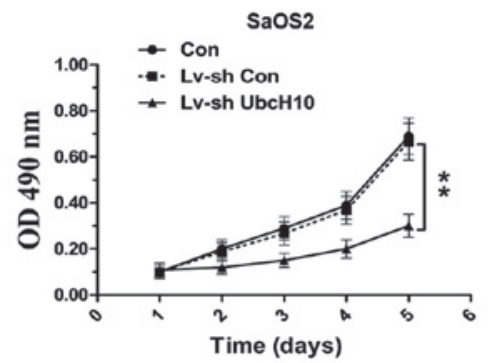

C

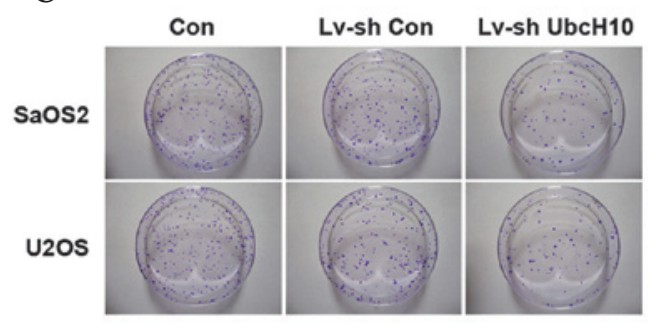

$\mathbf{E}$

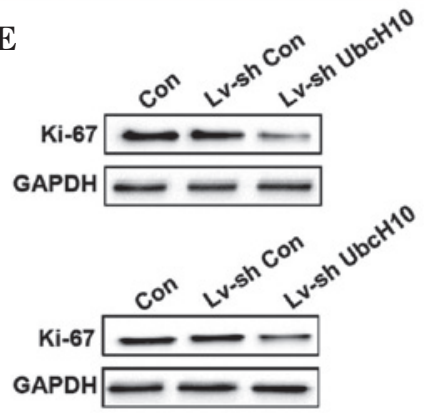

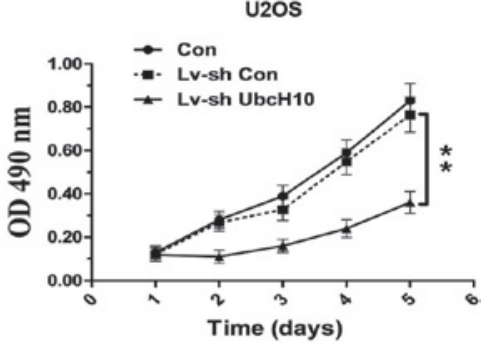

D

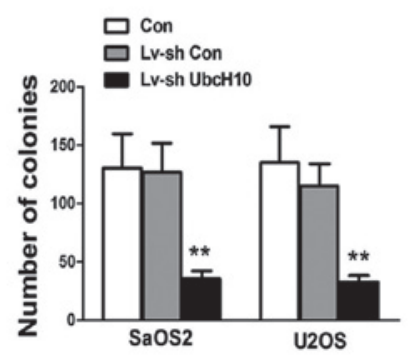

F

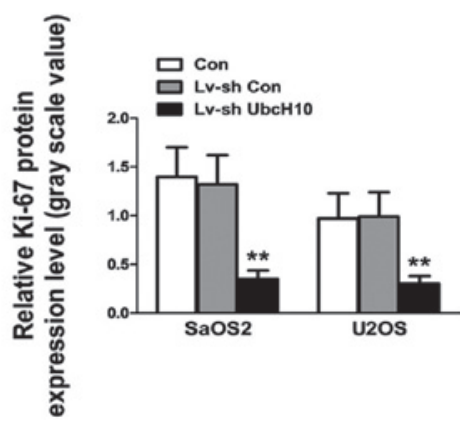

Figure 2. UbcH10-targeted RNA interference reduces osteosarcoma cellular proliferation and colony formation. Results of the MTT assay revealing the cellular proliferation of Con, and Lv-shCon- and Lv-shUbcH10-transfected (A) SaOS2 cells and (B) U2OS cells. (C) Representative images revealing the crystal violet-stained colonies of Con, and Lv-shCon- and Lv-shUbcH10-transfected SaOS2 and U2OS cells. (D) Calculation of colony numbers of Con, and Lv-shCon- and Lv-shUbcH10-transfected SaOS2 and U2OS cells. (E) Western blot analysis revealing the protein levels of Ki-67 in Con, and Lv-shCon- and Lv-shUbcH10-transfected SaOS2 and U2OS cells. GAPDH was used as a control protein. (F) Calculation of Ki-67 protein levels in SaOS2 and U2OS cells, normalized to GAPDH. ${ }^{* *} \mathrm{P}<0.01$ vs. the control group. Con, control; Lv-shCon, control small hairpin RNA-expressing lentivirus; Lv-shUbcH10, UbcH10-targeted small hairpin RNA-expressing lentivirus; OD, optical density.

colony formation ability is impaired in UbcH10 knock-down OS cells.

To confirm the results, western blotting was performed in order to analyze the expression level of the cellular proliferation marker, Ki-67. As shown in Fig. 2E and F, there was no significant difference in the protein level of Ki-67 in Lv-shCon-transfected U2OS and SaOS2 cells $(\mathrm{P}=0.657)$. By contrast, the expression of Ki-67 was markedly reduced in UbcH10 knock-down U2OS and SaOS2 cells $(\mathrm{P}=0.0035$ and $\mathrm{P}=0.0017$, respectively). These results indicate that fewer OS cells entered the process of proliferation following downregulation of $\mathrm{UbcH} 10$, a result is which is consistent with those of the MTT and colony formation assays.

UbcH10-targeted RNAi suppresses OS cell invasion and migration. The matrix invasion and migration abilities of cancer cells are closely associated with metastasis. Therefore, the effect of UbcH10 suppression on the invasion of OS cells was investigated. The results of the Transwell invasion assay revealed fewer UbcH10 knock-down U2OS and SaOS2 cells than control and Lv-shCon-transfected U2OS and $\mathrm{SaOS} 2$ cells in the lower chamber (Fig. 3A and B). The cell migration was investigated using a wound healing assay.
The quantification of cellular movement at $24 \mathrm{~h}$ revealed that cellular migration was significantly repressed in UbcH10 knock-down U2OS and SaOS2 cells compared with control and Lv-shCon-transfected cells $(\mathrm{P}=0.0029$ and $\mathrm{P}=0.0016$, respectively) (Fig. $3 \mathrm{C}$ and $\mathrm{D}$ ). These results suggest that the invasion and migration abilities of OS cells are impaired following knockdown of $\mathrm{UbcH} 10$.

UbcH10 knockdown of OS cells downregulates the expression of MMPs. The expression of MMPs in U2OS and SaOS2 cells was investigated. The levels of MMP-3 protein were significantly reduced in Lv-shUbcH10-transfected U2OS and $\mathrm{SaOS} 2$ cells compared with control and Lv-shCon-transfected cells ( $\mathrm{P}=0.0043$ and $\mathrm{P}=0.0061$, respectively) (Fig. 4A-C). Similar results were observed for MMP-9 (Fig. 4D-F). These results indicate that the MMP signaling pathway is disrupted in UbcH10 knockdown OS cells.

\section{Discussion}

$\mathrm{UbcH} 10$ is a potential cancer biomarker that is overexpressed in a variety of cancers. In order to investigate its functions in OS, UbcH10 was knocked down in the OS U2OS and SaOS2 cell lines. 

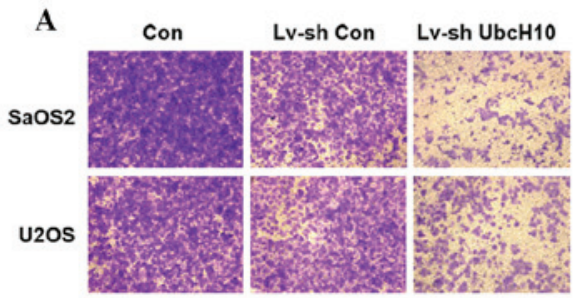

C

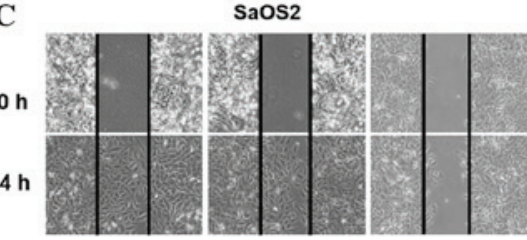

U2OS

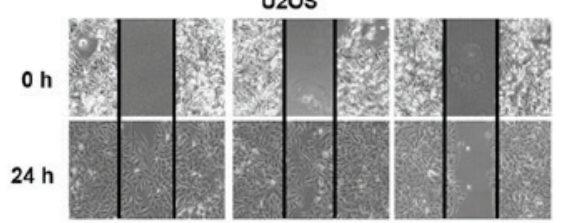

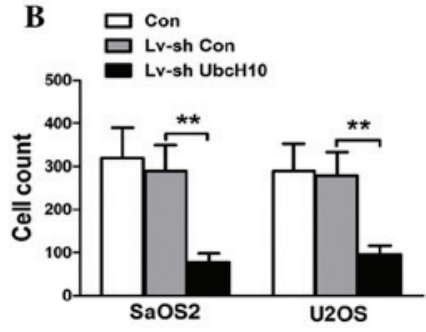

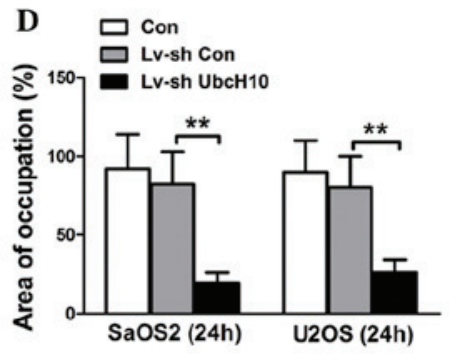

Figure 3. UbcH10-targeted RNA interference reduces osteosarcoma cell proliferation and colony formation. (A and B) MTT assay revealing the cellular proliferation of Con, and Lv-shCon- and Lv-shUbcH10-transfected SaOS2 and U2OS cells. ${ }^{* *} \mathrm{P}<0.01$ vs. the control group. (C) Representative images revealing crystal violet-stained colonies of Con, and Lv-shCon- and Lv-shUbcH10-transfected SaOS2 and U2OS cells. (D) Calculation of colony numbers of Con, and Lv-shCon- and Lv-shUbcH10-transfected SaOS2 and U2OS cells. ${ }^{* *} \mathrm{P}<0.01$ vs. the control group. Con, control; Lv-shCon, control small hairpin RNA-expressing lentivirus; Lv-shUbcH10, UbcH10-targeted small hairpin RNA-expressing lentivirus.

A

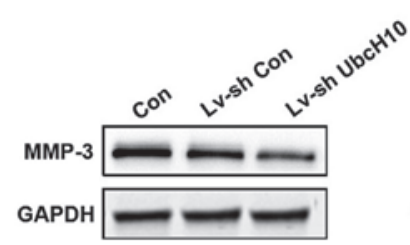

$\mathbf{C}$

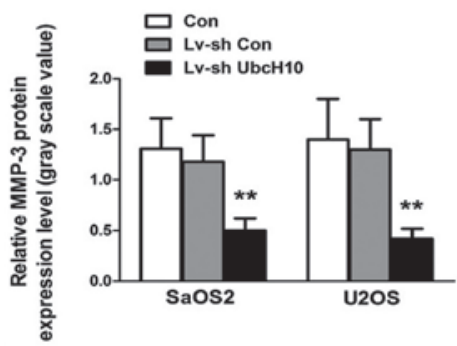

$\mathbf{E}$

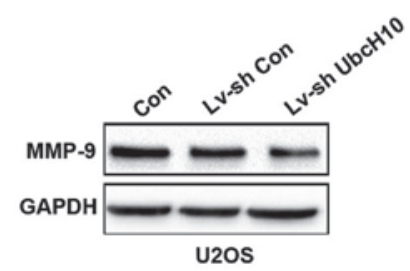

B

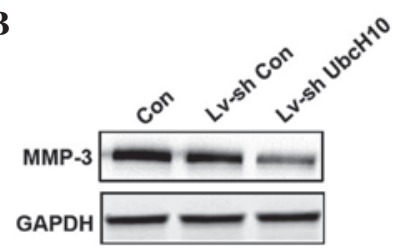

D

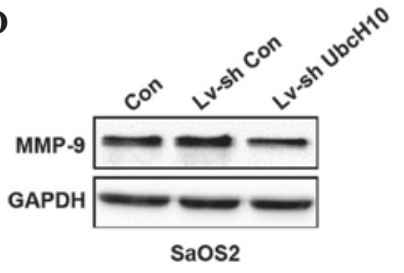

$\mathbf{F}$

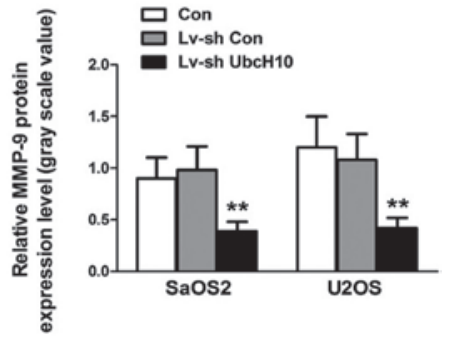

Figure 4. Protein levels of MMP-3 and -9 are downregulated in UbcH10 knock-down osteosarcoma cells. Western blot analysis showing the protein levels of MMP-3 in Con, and Lv-shCon- and Lv-shUbcH10-transfected (A) SaOS2 cells and (B) U2OS cells. GAPDH was used as a control protein. (C) Calculation of MMP-3 protein levels in SaOS2 and U2OS cells, normalized to GAPDH. ${ }^{* *} \mathrm{P}<0.01$ vs. the control group. Western blot analysis showing the protein levels of MMP-9 in Con, and Lv-shCon-and Lv-shUbcH10-transfected (C) SaOS2 cells and (D) U2OS cells. GAPDH was used as a control protein. (F) Calculation of MMP-9 protein levels in SaOS2 and U2OS cells, normalized to GAPDH. ${ }^{* *} \mathrm{P}<0.01$ vs. the control group. MMP, matrix metalloproteinase; Con, control; Lv-shCon, control small hairpin RNA-expressing lentivirus; Lv-shUbcH10, UbcH10-targeted small hairpin RNA-expressing lentivirus.

Western blot analysis revealed that the protein levels of $\mathrm{UbcH} 10$ in $\mathrm{Lv}$-shUbcH10 decreased to approximately one quarter of that in the control cells. This indicated that the recombinant lentivirus containing UbcH10-targeted shRNA could successfully 
knockdown UbcH10 in U2OS and SaOS2 cells. Lentiviruses are therefore useful for gene-targeted RNAi in OS cells in vitro.

The present study also identified that, as a result of decreased Ki-67 levels, a downregulation in the expression of UbcH10 inhibited cellular proliferation and colony formation in vitro. This confirmed that the expression of UbcH10 is correlated with the proliferation activity of cancer cells. In accordance with the results of the present study, a previous study demonstrated that a knockdown of UbcH10 inhibited the cellular proliferation of other cancer cells, including those of lung, glioma and colorectal cancers (13-15). It is therefore hypothesized that OS cell growth inhibition is caused by UbcH10-mediated cell cycle regulation. In order to address this, the present study analyzed the expression of the cellular proliferation marker, Ki-67 (16). Following UbcH10 knockdown, the decreased expression of Ki-67 indicated the presence of fewer dividing OS cells. This is consistent with its role in the regulation of mitotic exit and cell cycle progression through the destruction of mitosis-related substrates (5,7-9).

A downregulation in the expression of UbcH10 was also observed to impair the invasion and migration ability of OS cells. In lung cancer cells, UbcH10-targeted RNAi also inhibits cellular migration (15). In order to determine the underlying mechanisms involved in the impaired invasion and migration of UbcH10 knockdown OS cells, the expression of MMP-3 and MMP-9 was analyzed. The levels of MMP-3 and -9 proteins decreased significantly. MMPs are a family of zinc-dependent endopeptidases, which degrade proteins in the extracellular matrix (17), and are crucial in cancer cell invasion and migration $(17,18)$. Previous studies have demonstrated that an overexpression of MMP-3 in normal breast epithelium results in invasive tumor formation (19). Additionally, in a recent study, MMP-9 was identified as a potential biomarker for OS (20). The downregulation of MMP-3 and -9 confirms that tumorigenesis is inhibited in $\mathrm{UbcH} 10$ knockdown OS cells. However, whether MMP-3 and -9 are direct substrates of UbcH10-mediated ubiquitin-dependent proteolysis remains to be elucidated.

In conclusion, the results of the present study demonstrate that, via the deregulation of Ki-67 and MMPs, lentivirus-mediated UbcH10-targeted RNAi can lead to cell growth inhibition, decreased colony formation, and impaired cellular invasion and migration in the human OS U2OS and $\mathrm{SaOS} 2$ cell lines. These results indicate an important role for UbcH10 in OS progression, which suggests that UbcH10 may be a potential therapeutic target for the treatment of OS. Therapeutic strategies which target the UbcH10 gene or compounds that inhibit UbcH10 activity may be of use clinically for the treatment of OS and thus, further studies are required to investigate these methods.

\section{References}

1. Bacci G, Longhi A, Versari M, Mercuri M, Briccoli A and Picci P: Prognostic factors for osteosarcoma of the extremity treated with neoadjuvant chemotherapy: 15 -year experience in 789 patients treated at a single institution. Cancer 106: 1154-1161, 2006.

2. Ottaviani G and Jaffe N: The etiology of osteosarcoma. Cancer Treat Res 152: 15-32, 2009.

3. Mirabello L, Troisi RJ and Savage SA: Osteosarcoma incidence and survival rates from 1973 to 2004: data from the Surveillance, Epidemiology, and End Results Program. Cancer 115: 1531-1543, 2009.

4. Akiyama T, Dass CR and Choong PF: Novel therapeutic strategy for osteosarcoma targeting osteoclast differentiation, bone-resorbing activity, and apoptosis pathway. Mol Cancer Ther 7: 3461-3469, 2008

5. Townsley FM, Aristarkhov A, Beck S, Hershko A and Ruderman JV: Dominant-negative cyclin-selective ubiquitin carrier protein E2-C/UbcH10 blocks cells in metaphase. Proc Natl Acad Sci USA 94: 2362-2367, 1997.

6. Okamoto Y, Ozaki T, Miyazaki K, Aoyama M, Miyazaki M and Nakagawara A: UbcH10 is the cancer-related E2 ubiquitin-conjugating enzyme. Cancer Res 63: 4167-4173, 2003

7. Lin Y, Hwang WC and Basavappa R: Structural and functional analysis of the human mitotic-specific ubiquitin-conjugating enzyme, UbcH10. J Biol Chem 277: 21913-21921, 2002.

8. Aristarkhov A, Eytan E, Moghe A, Admon A, Hershko A and Ruderman JV: E2-C, a cyclin-selective ubiquitin carrier protein required for the destruction of mitotic cyclins. Proc Natl Acad Sci USA 93: 4294-4299, 1996.

9. Rape M, Reddy SK and Kirschner MW: The processivity of multiubiquitination by the APC determines the order of substrate degradation. Cell 124: 89-103, 2006.

10. Hao Z, Zhang $\mathrm{H}$ and Cowell J: Ubiquitin-conjugating enzyme UBE2C: molecular biology, role in tumorigenesis, and potential as a biomarker. Tumour Biol 33: 723-730, 2012.

11. Xie C,PowellC, Yao M, Wu J and Dong Q: Ubiquitin-conjugating enzyme E2C: a potential cancer biomarker. Int J Biochem Cell Biol 47: 113-117, 2014.

12. van Ree JH, Jeganathan KB, Malureanu L and van Deursen JM: Overexpression of the E2 ubiquitin-conjugating enzyme UbcH10 causes chromosome missegregation and tumor formation. J Cell Biol 188: 83-100, 2010.

13. Jiang L,Bao Y,LuoC, etal: Knockdown of ubiquitin-conjugating enzyme E2C/UbcH10 expression by RNA interference inhibits glioma cell proliferation and enhances cell apoptosis in vitro. J Cancer Res Clin Oncol 136: 211-217, 2010.

14. Chen SM, Jiang CY, Wu JY, et al: RNA interference-mediated silencing of UBCH10 gene inhibits colorectal cancer cell growth in vitro and in vivo. Clin Exp Pharmacol Physiol 37: 525-529, 2010.

15. Pallante P, Malapelle U, Berlingieri MT, et al: UbcH10 overexpression in human lung carcinomas and its correlation with EGFR and p53 mutational status. Eur J Cancer 49: 1117-1126, 2013.

16. Scholzen T and Gerdes J: The Ki-67 protein: from the known and the unknown. J Cell Physiol 182: 311-322, 2000.

17. Kleiner DE and Stetler-Stevenson WG: Matrix metalloproteinases and metastasis. Cancer Chemother Pharmacol 43 Suppl: S42-S51, 1999.

18. Davies KJ: The complex interaction of matrix metalloproteinases in the migration of cancer cells through breast tissue stroma. Int J Breast Cancer 2014: 839094, 2014.

19. Egeblad M and Werb Z: New functions for the matrix metalloproteinases in cancer progression. Nat Rev Cancer 2: 161-174, 2002.

20. Wang J, Shi Q, Yuan TX, et al: Matrix metalloproteinase 9 (MMP-9) in osteosarcoma: review and meta-analysis. Clin Chim Acta 433: 225-231, 2014. 